\title{
ON HERMITE-FEJÉR INTERPOLATION IN A JORDAN DOMAIN
}

\author{
CHARLES K. CHUI AND XIE-CHANG SHEN
}

\begin{abstract}
The Hermite-Fejér interpolation problem on a Jordan domain is studied. Under certain mild conditions on the smoothness of the boundary curve, we give both uniform and $L^{p}, 0<p<\infty$, estimates on the rate of convergence. Our estimates are sharp even for the unit disk setting.
\end{abstract}

\section{INTRODUCTION}

Let $D$ be a Jordan domain in the complex plane $\mathbb{C}$ with boundary $\Gamma$ and $z_{k}=z_{n k}, k=1, \ldots, n$, be sample points chosen on $\Gamma$. Also, let $q$ be a nonnegative integer and $N=N_{n}:=(q+1) n-1$. In this paper we will consider the interpolation problem:

$$
\widetilde{H}_{N}\left(f ; z_{k}\right)=f\left(z_{k}\right), \quad \widetilde{H}_{N}^{(j)}\left(f ; z_{k}\right)=a_{k}^{(j)},
$$

$k=1, \ldots, n$ and $j=1, \ldots, q$, where $f$ belongs to the class $A(\bar{D})$ of functions analytic in $D$ and continuous on $\bar{D}=D \cup \Gamma$, and $\widetilde{H}_{N}(f ; \cdot) \in \pi_{N}$, the space of all polynomials with degree at most $N$. Note that since $f$ is not necessarily differentiable at $z_{k}$ relative to $\bar{D}$ and the family of data values $\left\{a_{k}^{(j)}\right\}$ is arbitrarily given, the problem under consideration is different from the Hermite interpolation problem. In particular, by choosing $a_{k}^{(j)}=0$ for all $k=1, \ldots, q$, the problem

$$
H_{N}\left(f ; z_{k}\right)=f\left(z_{k}\right), \quad H_{N}^{(j)}\left(f ; z_{k}\right)=0,
$$

$k=1, \ldots, n$ and $j=1, \ldots, q$, where $f \in A(\bar{D})$ and $H_{N}(f ; \cdot) \in \pi_{N}$, is usually called the $(0,1, \ldots, q)$ Hermite-Fejér Interpolation Problem.

It is well known that even for the unit disk $U=\{z:|z|<1\}$, any $q$, and $z_{k}=e^{i 2 \pi k / n}$, there exists an $f \in A(\bar{U})$ such that $H_{N}(f ; \cdot)$ does not converge uniformly on $\bar{U}$ to $f$ (see [13]). In this paper, under certain smoothness conditions on the Jordan curve $\Gamma$, we will first give a necessary and sufficient

Received by the editors October 11, 1989.

1980 Mathematics Subject Classification (1985 Revision). Primary 41A05, 41A10, 41A25.

Key words and phrases. Hermite-Fejér interpolation, rate of convergence, Jordan domain, asymptotic formulas.

Research of the first author was supported in part by the National Science Foundation under Grant Nos. DMS-86-02337 and DMS 89-01345 and SDIO/IST managed by ARO under Contract No. DAAL 03-87-K-0025; research of the second author supported by NSFC and the Chinese National Commission of Education. 
condition on $f \in A(\bar{D})$ that guarantees uniform convergence of $\widetilde{H}_{N}(f ; \cdot)$ to $f$ on $\bar{D}$ for $a_{n}^{(j)}=o\left(n^{j} / \ln n\right)$, and derive the order of uniform convergence on $\bar{D}$ of the Hermite-Fejér interpolatory polynomials $H_{N}(f ; \cdot)$ to $f$ in terms of the modulus continuity of $f$. We will next show that for $a_{n}^{(j)}=o\left(n^{j}\right), \widetilde{H}_{N}(f ; \cdot)$ always converges in $L^{p}(\Gamma)$ to $f \in A(\bar{D}), 0<p<\infty$, and, in fact, a sharp order of convergence of $H_{N}(f ; \cdot)$ in $L^{p}(\Gamma), 0<p<\infty$, will be given.

Of course, for $q=0$, problems (1.1) and (1.2) become the Lagrange interpolation problem:

$$
L_{N}\left(f ; z_{k}\right)=f\left(z_{k}\right),
$$

$N=n-1, k=1, \ldots, n$, and $L_{N} \in \pi_{N}$. For an analytic Jordan curve $\Gamma$, Curtiss [3] has shown that $\left\|L_{N}(f ; \cdot)-f\right\|_{2} \rightarrow 0$ for all $f \in A(\bar{D})$ by using the Fejér nodes $z_{k}$ on $\Gamma$. Here and throughout, $\|\cdot\|_{p}$ denotes the $L^{p}$-norm on $\Gamma$. Later, for a Jordan curve $\Gamma$ of class $C^{2+\delta}$, for some $\delta>0, \mathrm{Al}^{\prime}$ per and Kalinogorskaja [2] improved the result in [3] by showing that

$$
\left\|L_{N}(f ; \cdot)-f\right\|_{p} \rightarrow 0
$$

for any $p, 0<p<\infty$. Recently, this result was further improved by the second author and Zhong [10] to a Jordan curve $\Gamma$ of class $C^{1+\delta}$ where the order of approximation $O\left(\omega\left(f ; \frac{1}{N}\right)\right)$ is also given. Here and throughout, $\omega(f ; \delta)$ denotes the modulus of continuity of $f$ on $\Gamma$ using the uniform norm. We remark that the $L^{p}, 0<p<\infty$, modulus of continuity cannot be used even for the $L^{p}$ estimate of $\left\|L_{N}(f ; \cdot)-f\right\|_{p}$.

The only result in the literature for Hermite-Féjer interpolation on a Jordan curve different from the circle was obtained by Gaier [6], where an analytic curve $\Gamma$ and $q=1$ are considered and the convergence is only uniform on compact subsets of $D$. Various recent results concerning convergence on the unit disk of Hermite-Féjer interpolatory polynomials at the $n$th roots of unity can be found in Szabados and Varma [11], Varma [12], and the second author $[8,9]$.

\section{MAIN RESUlTS}

Throughout this paper, $w=\Phi(z)$ denotes the exterior conformal map from $\mathbb{C} \backslash \bar{D}$ onto $|w|>1$ such that $\Phi(\infty)=\infty$ and $\Phi^{\prime}(\infty)>0$. Let $\Psi=\Phi^{-1}$ and write

$$
z=\Psi(w)=d w+a_{0}+a_{1} w^{-1}+\cdots,
$$

where $d=\Psi^{\prime}(\infty)>0$. It will be clear that by a standard transformation, we may assume, without loss of generality, that $d=1$. Extend $\Psi$ to a continuous function on $|w| \geq 1$ and set $z_{k}=z_{n k}=\Psi\left(w_{n k}\right)$ where $w_{n k}=w_{k}=e^{i 2 \pi k / n}$. Recall that the $z_{n k}$ 's are usually called the Fejér points on $\Gamma=\partial D$. We need some assumptions on the smoothness of $\Gamma$. 
Definition. (i) $\Gamma$ is said to be of class $j_{1}$ if $\Psi^{\prime}(w)$ exists and is continuous on $|w| \geq 1$, and its (uniform) modulus of continuity $\sigma_{1}(t)$ on $|w|=1$ satisfies the condition

$$
\int_{0}^{a} \frac{\sigma_{1}(t)}{t}|\ln t|^{2} d t<\infty, \quad a>0 .
$$

(ii) $\Gamma$ is said to be of class $j_{2}$ if $\Psi^{\prime \prime}(w)$ exists and is continuous on $|w| \geq 1$, and its (uniform) modulus of continuity $\sigma_{2}(t)$ on $|w|=1$ satisfies the condition

$$
\int_{0}^{a} \frac{\sigma_{2}(t)}{t}|\ln t| d t<\infty, \quad a>0 .
$$

It is well known [1] that if $\Gamma$ belongs to class $j_{1}$, then $\Psi$ satisfies:

$$
0<C_{1} \leq\left|\frac{\Psi(w)-\Psi(u)}{w-u}\right| \leq C_{2}
$$

for all $w \neq u$ and $|w|,|u| \geq 1$. We remark that in [1] it is shown that (2.4) already holds for those $\Gamma$ with

$$
\int_{0}^{a} \frac{\sigma_{1}(t)}{t} d t<\infty
$$

In addition, it is shown in the same paper that

$$
0<C_{1} \leq\left|\Psi^{\prime}(w)\right| \leq C_{2}
$$

for all $w,|w| \geq 1$.

Let

$$
\omega_{n}(z)=\prod_{j=1}^{n}\left(z-z_{j}\right) .
$$

Then for each $k,\left(z-z_{k}\right) / \omega_{n}(z)$ is analytic at $z_{k}$, so that we can write

$$
\left(\frac{z-z_{k}}{\omega_{n}(z)}\right)^{q+1}=\sum_{\nu=0}^{\infty} \alpha_{k \nu}\left(z-z_{k}\right)^{\nu}
$$

where $\alpha_{k \nu}=\alpha_{k \nu}(q, n), q=0,1, \ldots$ In the following, we will give an asymptotic estimate of $\alpha_{k \nu}=\alpha_{k \nu}(q, n)$ as $n \rightarrow \infty$. We need the notation

$$
\Omega_{n}(w)=\prod_{k=1}^{n} \frac{z-z_{k}}{w-w_{k}}, \quad z=\Psi(w) .
$$

Theorem 1. Let $\Gamma$ belong to class $j_{2}$. Then for each $\nu$ and $q=0,1, \ldots$,

$$
\alpha_{k \nu}=\alpha_{k \nu}(q, n)=O\left(\frac{1}{n^{q+1-\nu}}\right)
$$

and the estimate is uniform in $k, 1 \leq k \leq n$, as $n \rightarrow \infty$.

Here and throughout, $\sum_{l \neq k}$ denotes the summation over all $l=1, \ldots, n$ with $l \neq k$. To construct the interpolatory polynomials $\widetilde{H}_{N}(f ; \cdot)$ and $H_{N}(f ; \cdot)$ 
we introduce the fundamental functions:

$$
A_{k j}(z)=\left(\frac{\omega_{n}(z)}{z-z_{k}}\right)^{q+1} \frac{\left(z-z_{k}\right)^{j}}{j !} \sum_{\nu=0}^{q-j} \alpha_{k \nu}\left(z-z_{k}\right)^{\nu}
$$

where $j=0, \ldots, q$ and $l=1, \ldots, n$. It is obvious that $A_{k j} \in \pi_{N}$ and we will verify that they satisfy

$$
A_{k j}^{(\nu)}\left(z_{l}\right)=\delta_{k l} \delta_{\nu j}, \quad k, l=1, \ldots, n ; \nu, j=0, \ldots, q,
$$

where, as usual, $\delta_{i j}$ denotes the Kronecker delta.

Theorem 2. For any $f \in A(\bar{D})$, any nonnegative integer $q$, and arbitrary complex numbers $a_{k}^{(j)}, k=1, \ldots, n, j=1, \ldots, q$, there exists a unique $\widetilde{H}_{N}(f ; \cdot) \in \pi_{N}$ satisfying the interpolation conditions (1.1). Furthermore, $\widetilde{H}_{N}(f ; \cdot)$ is given by

$$
\widetilde{H}_{N}(f ; \cdot)=\sum_{k=1}^{n} f\left(z_{k}\right) A_{k 0}(\cdot)+\sum_{k=1}^{n} \sum_{j=1}^{q} a_{k}^{(j)} A_{k j}(\cdot) .
$$

In addition, under the assumption that $\Gamma$ belongs to the class $j_{2}$, the fundamental functions $A_{k j}$ satisfy the following estimates:

$$
\max _{z \in \bar{D}} \sum_{k=1}^{n}\left|A_{k j}(z)\right|=O\left(\frac{\ln n}{n^{j}}\right), \quad j=0, \ldots, q,
$$

and for $1<p<\infty$,

$$
\left\|\sum_{k=1}^{n} b_{k} A_{k j}(\cdot)\right\|_{p}=O\left(\frac{1}{n^{j}}\right) \max _{1 \leq k \leq n}\left|b_{k}\right|, \quad j=0, \ldots, q,
$$

for any sequence $\left\{b_{k}\right\}, k=1, \ldots, n$.

Of course, if we choose $a_{k}^{(j)}=0$, then the polynomials $\widetilde{H}_{N}(f ; \cdot)$ become $H_{N}(f ; \cdot)$ that satisfy the Hermite-Fejér interpolation condition (1.2). It is well known that even for the case $D=U$, the unit disk, there exists an $f \in A(\bar{U})$ such that $H_{N}(f ; \cdot)$ does not converge uniformly to $f$ on $\bar{U}$. We have the following result on the order of uniform approximation.

Theorem 3. Let $\Gamma$ belong to class $j_{2}$ and $f \in A(\bar{D})$. Then for any nonnegative integer $q$,

$$
\max _{z \in \bar{D}}\left|f(z)-H_{N}(f ; z)\right|=O\left(\omega\left(f ; \frac{1}{n}\right) \ln n\right) .
$$

We remark that this result is sharp as shown by the second author in [9] for $D=U$. For nonzero $a_{k}^{(j)}$, we have the following result. 
Theorem 4. Let $\Gamma$ belong to class $j_{2}, f \in A(\bar{D})$, and $q$ be any nonnegative integer. Suppose that

$$
\max _{1 \leq k \leq n}\left|a_{k}^{(j)}\right|=o\left(\frac{n^{j}}{\ln n}\right), \quad j=1, \ldots, q,
$$

and

$$
\lim _{\delta \rightarrow 0} \omega(f ; \delta) \ln \delta=0
$$

Then

$$
\lim _{N \rightarrow \infty} \max _{z \in \bar{D}}\left|f(z)-\widetilde{H}_{N}(f ; z)\right|=0 .
$$

For $L^{p}$ convergence, $0<p<\infty$, we no longer need $\ln n$ in (2.15) as in the following

Theorem 5. Let $\Gamma$ belong to class $j_{2}, f \in A(\bar{D}), q$ be any nonnegative integer, and $0<p<\infty$. Then

$$
\left\|f-H_{N}(f ; \cdot)\right\|_{p}=O\left(\omega\left(f ; \frac{1}{n}\right)\right) .
$$

Again, this result is sharp even for $D=U$ as shown in [10]. For nonzero $a_{k}^{(j)}$, we have the following result.

Theorem 6. Let $\Gamma$ belong to class $j_{2}, f \in A(\bar{D}), q$ be any nonnegative integer, and $\left\{a_{n}^{(j)}\right\}$ satisfy

$$
\max _{1 \leq k \leq n}\left|a_{k}^{(j)}\right|=o\left(n^{j}\right), \quad j=1, \ldots, q .
$$

Then

$$
\lim _{N \rightarrow \infty}\left\|f-\widetilde{H}_{N}(f ; \cdot)\right\|_{p}=0, \quad 0<p<\infty
$$

\section{Proof of Theorem 1}

To establish Theorem 1, we need three lemmas.

Lemma 1. Let $\Psi^{\prime \prime}$ be continuous on $|w| \geq 1$. Then for each $k=1, \ldots, n$,

$$
\omega_{n}^{\prime}\left(z_{k}\right)=n \frac{\Omega_{n}\left(w_{k}\right)}{\Psi^{\prime}\left(w_{k}\right) w_{k}}
$$

and

$$
\begin{aligned}
\sum_{l \neq k} \frac{1}{z_{k}-z_{l}} & =\frac{1}{2} \frac{\omega_{n}^{\prime \prime}\left(z_{k}\right)}{\omega_{n}^{\prime}\left(z_{k}\right)} \\
& =\frac{1}{2 \Psi^{\prime}\left(w_{k}\right)}\left[\frac{n-1}{w_{k}}+\frac{2 \Omega_{n}^{\prime}\left(w_{k}\right)}{\Omega_{n}\left(w_{k}\right)}-\frac{\Psi^{\prime \prime}\left(w_{k}\right)}{\Psi^{\prime}\left(w_{k}\right)}\right] .
\end{aligned}
$$

Proof. From (2.6) and (2.8), we have

$$
\omega_{n}(z)=\left(w^{n}-1\right) \Omega_{n}(w),
$$


so that

$$
\omega_{n}^{\prime}(z)=\left[n w^{n-1} \Omega_{n}(w)+\left(w^{n}-1\right) \Omega_{n}^{\prime}(w)\right] \frac{1}{\Psi^{\prime}(w)},
$$

from which (3.1) follows. To establish the two identities in (3.2), we first use logarithmic derivatives to obtain

$$
\frac{\beta_{k}^{\prime}(z)}{\beta_{k}(z)}=\sum_{l \neq k} \frac{1}{z-z_{l}}
$$

with $\beta_{k}(z):=\omega_{n}(z) /\left(z-z_{k}\right)$. Since $\beta_{k}\left(z_{k}\right)=w_{n}^{\prime}\left(z_{k}\right)$ and

$$
\begin{aligned}
\beta_{k}^{\prime}\left(z_{k}\right) & =\lim _{z \rightarrow z_{k}} \frac{\omega_{n}^{\prime}(z)\left(z-z_{k}\right)-\omega_{n}(z)}{\left(z-z_{k}\right)^{2}} \\
= & \lim _{z \rightarrow z_{k}} \frac{\omega_{n}^{\prime}(z)\left(z-z_{k}\right)-\left[\omega_{n}^{\prime}\left(z_{k}\right)\left(z-z_{k}\right)+\frac{\omega_{n}^{\prime \prime}\left(z_{k}\right)}{2}\left(z-z_{k}\right)^{2}+o\left(z-z_{k}\right)^{2}\right]}{\left(z-z_{k}\right)^{2}} \\
= & \lim _{z \rightarrow z_{k}} \frac{w_{n}^{\prime \prime}\left(z_{k}\right)\left(z-z_{k}\right)^{2}-\frac{w_{n}^{\prime \prime}\left(z_{k}\right)}{2}\left(z-z_{k}\right)^{2}+o\left(z-z_{k}\right)^{2}}{\left(z-z_{k}\right)^{2}}=\frac{1}{2} \omega_{n}^{\prime \prime}\left(z_{k}\right),
\end{aligned}
$$

we have established the first identity in (3.2). To derive the second identity in (3.2), we first observe that

$$
\begin{aligned}
\omega_{n}^{\prime \prime}\left(z_{k}\right)= & n(n-1) w_{k}^{-2} \Omega_{n}\left(w_{k}\right)+2 n w_{k}^{-1} \Omega_{n}^{\prime}\left(w_{k}\right) \frac{1}{\left[\Psi^{\prime}\left(w_{k}\right)\right]^{2}} \\
& -n w_{k}^{-1} \Omega_{n}\left(w_{k}\right) \frac{\Psi^{\prime \prime}\left(w_{k}\right)}{\left[\Psi^{\prime}\left(w_{k}\right)\right]^{3}}
\end{aligned}
$$

by using (3.3) and the fact that $w_{k}^{n}=1$. By substituting this quantity and the quantity in (3.1) into $\omega_{n}^{\prime \prime}\left(z_{k}\right) / \omega_{n}^{\prime}\left(z_{k}\right)$, we arrive at the second identity in (3.2).

In the following, we give certain estimates on $\Omega_{N}$ and its relation with $\Omega_{N}^{\prime}$. Lemma 2. If $\Gamma$ belongs to class $j_{1}$, then

$$
\max _{|w| \geq 1}\left|\Omega_{n}(w)-1\right|=o\left(\frac{1}{\ln n}\right) .
$$

Furthermore, if $\Gamma$ belongs to class $j_{2}$, then

$$
\max _{|w| \geq 1}\left|\frac{\Omega_{n}^{\prime}(w)}{\Omega_{n}(w)}\right|=o(1) .
$$

Proof. To prove (3.4), let

$$
g(w, u)= \begin{cases}(\Psi(w)-\Psi(u)) /(w-u) & \text { for } u \neq w \\ \Psi^{\prime}(w) & \text { for } u=w\end{cases}
$$


where $|u|,|w| \geq 1$. Hence, from the definition of $\Omega_{N}(w)$ and $g(w, u)$, we have

$$
\ln \Omega_{N}(w)=\sum_{k=1}^{n} \ln g\left(w, w_{k}\right),
$$

where the branch of the logarithm is taken so that $\ln 1=0$. On the other hand, it is clear that

$$
\frac{\partial \ln g(w, u)}{\partial u}=\frac{-\Psi^{\prime}(u)(w-u)+(\Psi(w)-\Psi(u))}{(w-u)(\Psi(w)-\Psi(u))}
$$

and

$$
\begin{aligned}
\left\|\Psi(w)-\Psi(u)-\Psi^{\prime}(u)(w-u)\right\| & =\left|\int_{\gamma}\left[\Psi^{\prime}(\xi)-\Psi^{\prime}(u)\right] d \xi\right| \\
& \leq C_{1} \sigma_{1}(|w-u|) \int_{\gamma}|d \xi| \leq C_{2}|w-u| \sigma_{1}(|w-u|),
\end{aligned}
$$

where $\gamma$ is a contour joining $u$ to $w$ on $|\xi| \geq 1$ with length bounded by $\frac{\pi}{2}|u-w|$ and $\sigma_{1}$ denotes the modulus of continuity of $\Psi^{\prime}$. By using (2.4), (3.8), and (3.9), we have

$$
\left|\frac{\partial \ln g(w, u)}{\partial u}\right| \leq C \frac{\sigma_{1}(|w-u|)}{|w-u|},
$$

for $|u|,|w| \geq 1$. Hence, from the hypothesis that $\Gamma$ belongs to class $j_{1}$, as a function of $u$ on $|u|=1$, the function $\ln g(w, u)$ satisfies the Dini condition uniformly on $|w|=1$. It follows that

$$
\ln g(w, u)=\sum_{j=1}^{\infty} \frac{a_{j}(w)}{u^{j}}
$$

uniformly on $|u|,|w| \geq 1$. From the property

$$
\sum_{k=1}^{n} w_{k}^{-j}= \begin{cases}0 & \text { if } n \nmid j \\ n & \text { if } n \mid j\end{cases}
$$

of the $n$th roots of unity, we have, from (3.7),

$$
\ln \Omega_{n}(w)=n \sum_{l=1}^{\infty} a_{l n}(w)
$$

uniformly on $|w| \geq 1$. To estimate $a_{j}(w)$, since $\Gamma$ belongs to class $j_{1}$ we may use the Hardy-Littlewood inequality (cf. [4, p. 100])

$$
\left|\Psi^{\prime \prime}(u)\right| \leq C \frac{\sigma_{1}(|u|-1)}{|u|-1}, \quad|u|>1 .
$$


Indeed, letting $1<\rho \leq \frac{3}{2}$, we have from (3.8), for $|w|=1$,

$$
\begin{aligned}
\int_{|u|=\rho} & \left|\frac{\partial^{2} \ln g(w, u)}{\partial u^{2}}\right||d u| \\
= & \int_{|u|=\rho}\left|\frac{\Psi^{\prime \prime}(u)}{\Psi(u)-\Psi(w)}+\frac{\left(\Psi^{\prime}(u)\right)^{2}}{(\Psi(u)-\Psi(w))^{2}}-\frac{1}{(w-u)^{2}}\right||d u| \\
\leq & \int_{|u|=\rho}\left|\frac{\Psi^{\prime \prime}(u)}{\Psi(n)-\Psi(w)}\right||d u| \\
& +\int_{|u|=\rho}\left|\frac{\Psi^{\prime}(u)}{\Psi(u)-\Psi(w)}-\frac{1}{w-u}\right|\left|\frac{\Psi^{\prime}(u)}{\Psi(u)-\Psi(w)}+\frac{1}{w-u}\right||d u| \\
& :=I_{1}+I_{2},
\end{aligned}
$$

where by applying (2.4) and (3.12), we have

$$
I_{1} \leq C_{1} \frac{\sigma_{1}(\rho-1)}{\rho-1} \int_{|u|=\rho} \frac{d u}{|w-u|} \leq C_{2} \frac{\sigma_{1}(\rho-1)}{\rho-1} \ln \frac{1}{\rho-1},
$$

and by using (3.9) and (2.4), we also have

$$
I_{2} \leq C_{1} \int_{|u|=\rho} \frac{\sigma_{1}(|w-u|)}{|w-u|^{2}}|d u| \leq C_{2} \frac{\sigma_{1}(\rho-1)}{\rho-1} \ln \frac{1}{\rho-1} .
$$

That is,

$$
\int_{|u|=\rho}\left|\frac{\partial^{2} \ln g(w, u)}{\partial u^{2}}\right||d u| \leq C \frac{\sigma_{1}(\rho-1)}{\rho-1} \ln \frac{1}{\rho-1}, \quad \rho>1 .
$$

By taking the second derivative of the power series (3.10) and applying the estimate in $(3.13)$, we have, for $j=2,3, \ldots$,

$$
\begin{aligned}
\left|a_{j}(w)\right| & =\left|\frac{1}{2 \pi i} \int_{|u|=\rho} \frac{1}{j(j+1)} \frac{\partial^{2} \ln g(w, u)}{\partial u^{2}} u^{j+1} d u\right| \\
& \leq \frac{\rho^{j+1}}{2 \pi j^{2}} \int_{|u|=\rho}\left|\frac{\partial^{2} \ln g(w, u)}{\partial u^{2}}\right||d u| \leq C \rho^{j+1} \frac{\sigma_{1}(\rho-1)}{j^{2}(\rho-1)} \ln \frac{1}{\rho-1} .
\end{aligned}
$$

By taking $\rho=1+\frac{1}{j}$, it follows that

$$
\max _{|w|=1}\left|a_{j}(w)\right| \leq C \frac{\sigma_{1}\left(j^{-1}\right)}{j} \ln j .
$$

We now apply this estimate to (3.11), yielding

$$
\begin{aligned}
\max _{|w|=1}\left|\ln \Omega_{n}(w)\right| & \leq C n \sum_{l=1}^{\infty} \frac{\sigma_{1}(1 / \ln )}{\ln } \ln (\ln ) \\
& \leq C \int_{n}^{\infty} \frac{\sigma_{1}(1 / t)}{t} \ln t d t=C \int_{0}^{1 / n} \frac{\sigma_{1}(s)}{s}|\ln s| d s \\
& \leq \frac{C}{\ln n} \int_{0}^{1 / n} \frac{\sigma_{1}(s)}{s}|\ln s|^{2} d s=o\left(\frac{1}{\ln n}\right),
\end{aligned}
$$


where (2.2) has been used. This estimate is equivalent to (3.4).

To prove (3.5) for $\Gamma$ belonging to class $j_{2}$, we will apply the inequality of Hardy-Littlewood

$$
\left|\Psi^{\prime \prime \prime}(u)\right| \leq C \frac{\sigma_{2}(|u|-1)}{|u|-1}, \quad|u|>1,
$$

where $\sigma_{2}$ denotes the modulus of continuity of $\Psi^{\prime \prime}(u)$. Set

$$
g_{1}(w, u)=\frac{\Psi^{\prime}(u)}{\Psi(w)-\Psi(u)}-\frac{1}{w-u} .
$$

Then by taking the logarithmic derivative of $\Omega_{n}$ and $g\left(w, w_{k}\right)$ in (2.8) and (3.6), respectively, we have

$$
\frac{\Omega_{n}^{\prime}(w)}{\Omega_{n}(w)}=\sum_{k=1}^{n} \frac{g^{\prime}\left(w, w_{k}\right)}{g\left(w, w_{k}\right)}=\sum_{k=1}^{n} g_{1}\left(w, w_{k}\right),
$$

where $g_{1}(w, u)=\Psi^{\prime}(u) /(\Psi(w)-\Psi(u))-1 /(w-u)$, hence, in view of $\Gamma$ belonging to class $j_{2}$, we have

$$
g_{1}(w, u)=\sum_{j=1}^{\infty} \frac{b_{j}(w)}{u^{j}}
$$

uniformly on $|u|,|w| \geq 1$. By applying (3.14) we may obtain an estimate similar to that of (3.13), namely,

$$
\int_{|u|=\rho}\left|\frac{\partial^{2} g_{1}(w, u)}{\partial u^{2}}\right||d u| \leq C \frac{\sigma_{2}(\rho-1)}{\rho-1} \ln \frac{1}{\rho-1},
$$

where $\rho>1$. Hence, as before, we have

$$
\max _{|w|=1}\left|b_{j}(w)\right| \leq C \frac{\sigma_{2}\left(j^{-1}\right)}{j} \ln j
$$

and

$$
\frac{\Omega_{n}^{\prime}(w)}{\Omega_{n}(w)}=n \sum_{l=1}^{\infty} b_{l n}(w), \quad|w|=1
$$

so that

$$
\max _{|w| \geq 1}\left|\frac{\Omega_{n}^{\prime}(w)}{\Omega_{n}(w)}\right| \leq C n \sum_{l=1}^{\infty} \frac{\sigma_{2}(1 / \ln )}{\ln } \ln (\ln ) \leq \int_{0}^{1 / n} \frac{\sigma_{2}(t)}{t}|\ln t| d t
$$

which is $o(1)$ by (2.3). This completes the proof of the lemma.

Remark. In [10], where Lagrange interpolation (or $q=0$ ) was considered, the Jordan curve $\Gamma$ was assumed to belong to $C^{1+\delta}, \delta>0$. However, from our estimate (3.4) and the procedure in [10], it can be shown that the result there also holds for $\Gamma$ belonging to class $j_{1}$.

As a consequence of estimates (3.5) in Lemma 2, the identity (3.2) in Lemma 1 yields the following result. 
Corollary 1. Let $\Gamma$ belong to class $j_{2}$. Then

$$
\sum_{l \neq k} \frac{1}{z_{k}-z_{l}}=\frac{(n-1)}{2 w_{k} \Psi^{\prime}\left(w_{k}\right)}-\frac{\Psi^{\prime \prime}\left(w_{k}\right)}{2\left[\Psi^{\prime}\left(w_{k}\right)\right]^{2}}+o(1)
$$

uniformly in $k, 1 \leq k \leq n$.

In the proof of Theorem 1, the following estimates will also be used.

Lemma 3. Let $\Gamma$ belong to $j_{2}$. Then for $k=1,2, \ldots, n$ and $r=0,1, \ldots$

$$
\sum_{l \neq k} \frac{1}{\left(z_{k}-z_{l}\right)^{r+1}}=O\left(n^{r+1}\right)
$$

and

$$
\left.\frac{d^{r}}{d z^{r}}\left(\frac{z-z_{k}}{\omega_{n}(z)}\right)\right|_{z=z_{k}}=O\left(n^{r-1}\right)
$$

uniformly in $k, 1 \leq k \leq n$.

Proof. The estimate (3.18) for $r=0$ can easily be deduced by (3.17). For $r \geq 1$, by using $(2.4)$ we have

$$
\begin{aligned}
\left|\sum_{l \neq k} \frac{1}{\left(z_{k}-z_{l}\right)^{r+1}}\right| & \leq \sum_{l \neq k}\left|\frac{1}{z_{k}-z_{l}}\right|^{r+1} \leq C_{2} \sum_{l \neq k}\left|\frac{1}{w_{k}-w_{l}}\right|^{r+1} \\
& \leq 2 C_{2} \sum_{l=1}^{[(n-1) / 2]} \frac{1}{(2 \sin l \pi / n)^{r+1}} \\
& \leq 2 C_{2} \sum_{l=1}^{[(n-1) / 2]} \frac{1}{(4 l / n)^{r+1}}=O\left(n^{r+1}\right) .
\end{aligned}
$$

We are going to verify (3.19) by induction. For $r=0$, by using (3.1), (3.4), and (2.5), we have (3.19). For $r \geq 1$, by the induction hypothesis and using (3.18), we obtain

$$
\begin{aligned}
& \left.\left(\frac{z-z_{k}}{\omega_{n}(z)}\right)^{(s+1)}\right|_{z=z_{k}}=-\left.\left(\frac{z-z_{k}}{\omega_{n}(z)} \sum_{\substack{l=1 \\
l \neq k}}^{n} \frac{1}{z-z_{l}}\right)^{(s)}\right|_{z=z_{k}} \\
& =-\left.\sum_{i=0}^{s}\left(\begin{array}{l}
s \\
\nu
\end{array}\right)\left(\frac{z-z_{k}}{\omega_{n}(z)}\right)^{(\nu)}\left(\sum_{\substack{l=1 \\
l \neq k}}^{n} \frac{1}{z-z_{l}}\right)^{(s-l)}\right|_{z=z_{k}} \\
& =-\left.\sum_{v=0}^{s}\left(\begin{array}{l}
s \\
\nu
\end{array}\right)\left(\frac{z-z_{k}}{\omega_{n}(z)}\right)^{(\nu)} \sum_{\substack{l=1 \\
l \neq k}}^{n} \frac{(-1)^{s-\nu}(s-\nu) !}{\left(z-z_{l}\right)^{s-\nu+1}}\right|_{z=z_{k}} \\
& =\sum_{v=0}^{n}\left(\begin{array}{l}
s \\
\nu
\end{array}\right) O\left(n^{n-1}\right) \cdot O\left(n^{s-\nu+1}\right)=O\left(n^{s}\right) .
\end{aligned}
$$

This completes the proof of the lemma. 
We are now ready to prove Theorem 1 .

Proof of Theorem 1. For $q=0$, from (2.7), it is clear that

$$
\sigma_{k \nu}(0)=\left.\frac{1}{\nu !} \frac{d^{\nu}}{d z^{\nu}}\left(\frac{z-z_{k}}{\omega_{n}(z)}\right)\right|_{z=z_{k}}
$$

which yields (2.9) by using (3.19) in Lemma 3. We will now use induction in $q$. Indeed, by the induction hypothesis and using (3.19) in Lemma 3 it follows that

$$
\begin{aligned}
\sigma_{k \nu}(q+1) & =\left.\frac{1}{\nu !} \frac{d^{\nu}}{d z^{\nu}}\left(\frac{z-z_{k}}{\omega_{n}(z)}\right)^{q+2}\right|_{z=z_{k}} \\
& =\left.\frac{1}{\nu !} \sum_{j=0}^{\nu}\left(\begin{array}{c}
\nu \\
j
\end{array}\right) \frac{d^{j}}{d z^{j}}\left(\frac{z-z_{k}}{\omega_{n}(z)}\right)^{q+1} \frac{d^{\nu-j}}{d z^{\nu-j}}\left(\frac{z-z_{k}}{\omega_{n}(z)}\right)\right|_{z=z_{k}} \\
& =\frac{1}{\nu !} \sum_{j=0}^{\nu}\left(\begin{array}{c}
\nu \\
j
\end{array}\right) j ! O\left(\frac{1}{n^{q-j+1}}\right) O\left(n^{\nu-j-1}\right)=O\left(\frac{1}{n^{q+2-\nu}}\right) .
\end{aligned}
$$

This completes the proof of Theorem 1 .

\section{Proof of TheOREM 2}

We first establish the existence and uniqueness of $\widetilde{H}_{N}(f ; \cdot)$ for any given $f \in A(\bar{D})$. Since $N=(q+1) n-1$, it follows from the definition (2.11) that $A_{k_{j}} \in \pi_{N}$. Hence, $\widetilde{H}_{N}(f ; \cdot) \in \pi_{N}$ also. Next, we will establish (2.11). For $l \neq k$, it is clear from the first factor that $A_{k j}^{(\nu)}\left(z_{l}\right)=0$ for all $\nu, j=0, \ldots, q$. We now consider the case $l=k$. From (2.10) and (2.7), it follows that

$$
\begin{aligned}
A_{k j}(z) & =\left(\frac{\omega_{n}(z)}{z-z_{k}}\right)^{q+1} \frac{\left(z-z_{k}\right)^{j}}{j !}\left[\left(\frac{z-z_{k}}{\omega_{n}(t)}\right)^{q+1}-\sum_{\mu=q-j+1}^{\infty} \alpha_{k \mu}\left(z-z_{k}\right)^{\mu}\right] \\
& =\frac{\left(z-z_{k}\right)^{j}}{j !}-\left(\frac{\omega_{n}(z)}{z-z_{k}}\right)^{q+1} \frac{1}{j !} \sum_{\mu=q-j+1}^{\infty} \alpha_{k \mu}\left(z-z_{k}\right)^{\mu+j} .
\end{aligned}
$$

Hence, for $\nu<j$, we have $A_{k j}^{(\nu)}\left(z_{k}\right)=0$. For $\nu=j$, then $A_{k j}^{(j)}\left(z_{k}\right)=1$. Finally, for $\nu>j$, we also have $A_{k j}^{(\nu)}\left(z_{k}\right)=0$. This establishes the interpolatory property of $A_{k j}$ in (2.11). Thus, by defining $\widetilde{H}_{N}(f ; \cdot)$ as in $(2.12)$, $\widetilde{H}_{N}(f ; \cdot)$ solves the interpolation problem $(1.1)$. The uniqueness of $\widetilde{H}_{N}(f ; \cdot)$ is trivial.

In order to establish the estimates (2.13) and (2.14), we need the following lemma. 
Lemma 4. Let $\Gamma$ belong to class $j_{1}$. Then

$$
\begin{gathered}
\max _{z \in \bar{D}} \sum_{k=1}^{n}\left|\frac{\omega_{n}(z)}{\left(z-z_{k}\right) \omega_{n}^{\prime}\left(z_{k}\right)}\right|=O(\ln n), \\
\max _{z \in \bar{D}} \sum_{k=1}^{n}\left|\frac{\omega_{n}(z)}{\left(z-z_{k}\right) \omega_{n}^{\prime}\left(z_{k}\right)}\right|^{1+\delta}=O(1), \quad \delta>0,
\end{gathered}
$$

and for $1<p<\infty$,

$$
\left\|\sum_{k=1}^{n} b_{k} \frac{\omega_{n}(z)}{\left(z-z_{k}\right) \omega_{n}^{\prime}\left(z_{k}\right)}\right\|_{p}=O\left(\max _{1 \leq k \leq n}\left|b_{k}\right|\right) .
$$

Proof. From (2.8) and (3.1), we have

$$
\frac{\omega_{n}(z)}{\left(z-z_{k}\right) \omega_{n}^{\prime}\left(z_{k}\right)}=\frac{w_{k} \Psi^{\prime}\left(w_{k}\right)\left(w^{n}-1\right)}{n\left(\Psi(w)-\Psi\left(w_{k}\right)\right)} \frac{\Omega_{n}(w)}{\Omega_{n}\left(w_{k}\right)},
$$

where $z=\Psi(w)$ and $z_{k}=\Psi\left(w_{k}\right)$. For the unit disk, it is well known (cf. Gaier [6, pp. 80-81]) that

$$
\max _{|w| \leq 1} \sum_{k=1}^{n}\left|\frac{w_{k}}{n} \frac{w^{n}-1}{w-w_{k}}\right|=O(\ln n) .
$$

In addition, for $\delta>0$ it is also well known (cf. [9]) that

$$
\max _{|w| \leq 1} \sum_{k=1}^{n}\left|\frac{w_{k}}{n} \frac{w^{n}-1}{w-w_{k}}\right|^{1+\delta}=O(1) .
$$

Hence, by applying (2.4), (2.5), and (3.4) in Lemma 2 to (4.4), we have both (4.1) and (4.2). Next, by (4.4), it follows that

$$
\begin{aligned}
\sum_{k=1}^{n} b_{k} & \frac{\omega_{n}(z)}{\left(z-z_{k}\right) \omega_{n}^{\prime}\left(z_{k}\right)} \\
= & \sum_{k=1}^{n} b_{k} \frac{w_{k} \Psi^{\prime}\left(w_{k}\right)\left(x^{n}-1\right)}{n\left(\Psi(w)-\Psi\left(w_{k}\right)\right)}\left(\frac{\Psi_{n}(w)}{\Psi_{n}\left(w_{k}\right)}-1\right)+\sum_{k=1}^{n} b_{k} \frac{w_{k}}{n} \frac{w^{n}-1}{w-w_{k}} \\
& +\sum_{k=1}^{n} b_{k} \frac{w_{k}}{n}\left(w^{n}-1\right)\left[\frac{\Psi^{\prime}\left(w_{k}\right)}{\Psi(w)-\Psi\left(w_{k}\right)}-\frac{1}{w-w_{k}}\right] \\
:= & I_{5}+I_{6}+I_{7} .
\end{aligned}
$$

By applying (4.1) and (3.4) in Lemma 2, we obtain

$$
\max _{z \in \bar{D}}\left|I_{5}\right|=O\left(\max _{1 \leq k \leq n}\left|b_{k}\right|\right) \text {. }
$$

In order to estimate $I_{7}$, we may assume, without loss of generality, that $|w-1|$ is not greater than $\left|w-w_{k}\right|, k=1,2, \ldots, n-1$, so that

$$
|\arg w| \leq \frac{\pi}{n}, \quad\left|\frac{w^{n}-1}{w-1}\right| \leq n,
$$


and

$$
\left|\frac{1}{w-w_{k}}\right| \leq \begin{cases}\pi / n, & 1 \leq k \leq n / 2 \\ (n-k) / k, & n / 2<k \leq n-1\end{cases}
$$

Hence from (3.9), we have

$$
\left|b_{n} \frac{w_{n}}{n}\left(w^{n}-1\right)\left[\frac{\Psi(1)}{\Psi(w)-\Psi(1)}-\frac{1}{w-1}\right]\right| \leq C\left|b_{n}\right|
$$

and

$$
\begin{aligned}
& \left|\sum_{k=1}^{n-1} b_{k} \frac{w_{k}}{n}\left(w^{n}-1\right)\left[\frac{\Psi^{\prime}\left(w_{k}\right)}{\Psi(w)-\Psi\left(w_{k}\right)}-\frac{1}{w-w_{k}}\right]\right| \\
& \leq C \max _{1 \leq k \leq n-1}\left|b_{k}\right| \frac{1}{n} \sum_{k=1}^{n-1} \frac{\sigma_{1}\left(\left|w-w_{k}\right|\right)}{\left|w-w_{k}\right|} \\
& \leq C \max _{1 \leq k \leq n-1}\left|b_{k}\right| \frac{1}{n} \sum_{k=1}^{n} \frac{\sigma_{1}(k / n)}{k / n} \\
& \leq C \max _{1 \leq k \leq n-1}\left|b_{k}\right| \int_{0}^{1} \frac{\sigma_{1}(t)}{t} d t \leq C_{1 \leq k \leq n-1}\left|b_{k}\right|,
\end{aligned}
$$

where the condition in (2.2) is used. Thus combining (4.5) and (4.6), we obtain

$$
\max _{|z| \in \bar{D}}\left|I_{7}\right|=O\left(\max _{1 \leq k \leq n}\left|b_{k}\right|\right) \text {. }
$$

Finally, by the Marcinkiewicz-Zygmund inequality for $1<p<\infty$ (cf. [15]), we have

$$
\left\|I_{6}\right\|_{p}=O\left(\frac{1}{n} \sum_{k=1}^{n}\left|b_{k}\right|^{p}\right)^{1 / p}=O\left(\max _{1 \leq k \leq n}\left|b_{k}\right|\right) .
$$

This completes the proof of the lemma.

We now return to the estimates of (2.13) and (2.14) and obtain

$$
\begin{aligned}
A_{k j}(z)= & \left(\frac{\omega_{n}(z)}{\left(z-z_{k}\right)}\right)^{q+1} \frac{1}{j !} \alpha_{k q-j}\left(z-z_{k}\right)^{q} \\
& +\left(\frac{\omega_{n}(z)}{z-z_{k}}\right)^{q+1} \frac{\left(z-z_{k}\right)^{j}}{j !} \sum_{\nu=0}^{q-j-1} \alpha_{k \nu}\left(z-z_{k}\right)^{\nu} \\
& :=I_{8}(z)+I_{9}(z) .
\end{aligned}
$$

Hence, by (3.1) in Lemma 1, (3.4) in Lemma 2, (2.5), (2.9) in Theorem 1, and (4.1), (4.3) in Lemma 4, we have

$$
\sum_{k=1}^{n}\left|I_{8}(z)\right|=O\left(\frac{1}{n^{j}}\right) \max _{z \in \bar{D}} \sum_{k=1}^{n}\left|\frac{\omega_{n}(z)}{\left(z-z_{k}\right) \omega_{n}^{\prime}\left(z_{k}\right)}\right|=O\left(\frac{\ln n}{n^{j}}\right)
$$

and for $1<p<+\infty$

$$
\left\|\sum_{k=1}^{n} b_{k} I_{q}(z)\right\|_{j}=O(n) \max _{1 \leq k \leq n}\left|b_{k} \alpha_{k q-j}\right|=O\left(\frac{1}{n^{j}}\right) \max _{1 \leq k \leq n}\left|b_{k}\right| .
$$


Similarly, by (3.1) in Lemma 1, (3.4) in Lemma 2, (2.5), (2.9) in Theorem 1, and $(4.2)$ in Lemma 4 , we have

$$
\sum_{k=1}^{n}\left|I_{9}(z)\right|=O\left(\frac{1}{n^{j}}\right) .
$$

By combining these estimates, we have established both (2.13) and (2.14).

\section{Proof of Theorems $3-6$}

Let $\Gamma$ belong to class $j_{2}$ and $f \in A(\bar{D})$. It is known (cf. Theorems 1 and 6 in [5, Chapter 9]) that there exists $P_{N} \in \pi_{N}$ such that

$$
\max _{z \in \bar{D}}\left|f(z)-P_{N}(z)\right|=O\left(\omega\left(f ; \frac{1}{N}\right)\right)
$$

and

$$
\max _{z \in \bar{D}}\left|P_{N}^{(m)}(z)\right|=O\left(N^{m} \omega\left(f ; \frac{1}{N}\right)\right), \quad m=1,2, \ldots
$$

By using the first part of Theorem 2, we have

$$
\begin{aligned}
f(z)-H_{N}(f ; z)= & f(z)-P_{N}(z)+\sum_{k=1}^{N}\left(P_{N}\left(z_{k}\right)-f\left(z_{k}\right)\right) A_{k 0}(z) . \\
& +\sum_{k=1}^{n} \sum_{j=1}^{q} P_{N}^{(j)}\left(z_{k}\right) A_{k j}(z)
\end{aligned}
$$

Hence, by applying (2.13) and (2.14) of Theorem 2 and (5.1), (5.2) above, we have completed the proof of Theorem 3 . Next, we write

$$
f(z)-\widetilde{H}_{N}(f ; z)=f(z)-H_{N}(f ; z)+\sum_{k=1}^{N} \sum_{j=1}^{q} a_{k}^{(j)} A_{k j}(z) .
$$

Here, by using the hypothesis (2.17) and Theorem 3, we have

$$
\max _{z \in \bar{D}}\left|f(z)-H_{N}(f ; z)\right| \rightarrow 0,
$$

and by using the hypothesis (2.16) and applying (2.13) in Theorem 2, we also have

$$
\max _{z \in \bar{D}}\left|\sum_{k=1}^{N} \sum_{j=1}^{q} a_{k}^{(j)} A_{k j}(z)\right| \rightarrow 0 .
$$

This completes the proof of Theorem 4. The proofs of Theorems 5 and 6 are similar simply by applying (2.14) in Theorem 2, noting that Hölder's inequality can be applied for $0<p \leq 1$ and using the result for $p=2$.

\section{Final Remarks}

In this section, we give examples of the domain $D$ whose boundary curve $\Gamma$ belongs to classes $j_{1}$ and $j_{2}$. Let $\Gamma$ be of class $C^{1}$ and denote its angle of inclination as a function of arc length $s$ by $\theta(s), 0 \leq s \leq|\Gamma|$, the length of $\Gamma$. 
Proposition 1. If $\Gamma$ satisfies

$$
\int_{0}^{a} \frac{\omega(\theta ; t)}{t}|\ln t|^{3} d t<\infty, \quad a>0,
$$

then $\Gamma$ belongs to class $j_{1}$.

Of course, every $\Gamma$ of class $C^{1+\delta}$ for some $\delta>0$ satisfies (6.1). We also have the following

Proposition 2. If $\Gamma$ satisfies

$$
\int_{0}^{a} \frac{\omega\left(\theta^{\prime} ; t\right)}{t}|\ln t|^{2} d t<\infty, \quad a>0,
$$

then $\Gamma$ belongs to class $j_{2}$.

Of course, every $\Gamma$ of class $C^{2+\delta}$ for some $\delta>0$ satisfies (6.2).

To prove these results, we need the following result in [14]: If

$$
\int_{0}^{a} \frac{\omega\left(\theta^{(n)} ; t\right)}{t} d t<\infty, \quad a>0,
$$

then $\Psi^{(n+1)}$ is continuous on $|w| \geq 1$ and

$$
\begin{aligned}
\omega\left(\Psi^{(n+1)} ; t\right)=O\left(\int_{0}^{t} \frac{\omega\left(\theta^{(n)} ; \tau\right)}{\tau} d \tau\right. \\
\left.\quad+t \int_{t}^{a} \frac{\omega\left(\theta^{(n)} ; \tau\right)}{\tau^{2}} d \tau+t \ln \frac{1}{t}\right), \quad a>0 .
\end{aligned}
$$

Let $n=0$. If $(6.1)$ is satisfied, so is $(6.3)$, and hence $\Psi^{\prime}$ is continuous on $|w| \geq 1$. Using (6.4) for $n=0$, we have

$$
\begin{aligned}
& \int_{0}^{a} \frac{\omega\left(\Psi^{\prime} ; t\right)}{t}|\ln t|^{2} d t \\
& =O\left(\int_{0}^{a}\left(\int_{0}^{t} \frac{\omega(\theta ; \tau)}{\tau} d \tau\right) \frac{|\ln t|^{2}}{t} d t\right. \\
& \left.\quad \quad \quad \quad \int_{0}^{a}\left(t \int_{t}^{a} \frac{\omega(\theta ; \tau)}{\tau^{2}} d \tau\right) \frac{|\ln t|^{2}}{t} d t+\int_{0}^{a} \frac{t \ln (1 / t)}{t}|\ln t|^{2} d t\right) \\
& =O\left(\int_{0}^{a}\left(\int_{\tau}^{a} \frac{|\ln t|^{2}}{t} d t\right) \frac{\omega(\theta ; \tau)}{\tau} d \tau\right. \\
& \left.\quad+\int_{0}^{a}\left(\int_{0}^{\tau}|\ln t|^{2} d t\right) \frac{\omega(\theta ; \tau)}{\tau^{2}} d \tau\right)+O(1) \\
& =O\left(\int_{0}^{a} \frac{\omega(\theta ; t)}{t}|\ln t|^{3} d t\right)+O(1)<\infty .
\end{aligned}
$$


That is, $\Gamma$ is of class $j_{1}$. This completes the proof of Proposition 1. The proof of Proposition 2 is similar by applying $n=1$ in (6.3) and (6.4), using the condition (6.2).

We conclude this paper by posing three open problems.

(1) In this paper, we consider the $(0,1, \ldots, q)$ Hermite-Fejér interpolation problem where the interpolatory polynomials $H_{N}(f ; \cdot)$ satisfy $H_{N}^{(j)}\left(f ; z_{k}\right)=$ $0, j=1, \ldots, q$ and $k=1, \ldots, n$. It is interesting to study if the convergence and estimates in this paper are still valid if we impose a more general interpolatory condition:

$$
H_{N}^{(j)}\left(f ; z_{k}\right)=0 \text { for } j=1, \ldots, q_{k}, k=1, \ldots, n,
$$

where $q_{k}=q_{k}(n)$ satisfies $\max _{1 \leq k \leq n} q_{k}(n) \leq M<\infty$ for all $n$.

(2) How much can the Fejér points $z_{k}=z_{n k}$ be perturbed on $\Gamma$ so that the convergence and estimates in this paper are still valid?

(3) If $D$ is different from the unit disk, do there exist $\left(0, m_{1}, \ldots, m_{q}\right)$ Birkhoff-Fejér interpolants $B_{N}(f ; \cdot)$; that is,

$$
B_{N}\left(f ; z_{k}\right)=f\left(z_{k}\right) \text { and } B_{N}^{\left(m_{j}\right)}\left(f ; z_{k}\right)=0 \text {, }
$$

for $j=1, \ldots, q$ and $k=1, \ldots, n$ ? If $B_{N}(f ; \cdot)$ exist, do they converge to $f$ in $L^{p}, 0<p<\infty$ ? For the unit disk, results on convergence and estimates have been obtained in [10].

\section{REFERENCES}

1. S. Ya. $\mathrm{Al}^{\prime}$ per, On the approximation in the mean to analytic functions in classes $E_{p}$, Investigation of Modern Problems of Theory of Functions of a Complex Variable, Gosudarstv. Izdat. Fiz.-Mat. Lit., Moscow, 1960, pp. 273-286.

2. S. Ya. Al' per and G. I. Kalinogorskaja, The convergence of Lagrange interpolation on polynomials in the complex domain, Izv. Vyš̌. Učebn. Zaved. Mat. 1969, no. 11(60), 13-23.

3. J. H. Curtiss, Convergence of complex Lagrange interpolation polynomials on the locus of the interpolation points, Duke Math. J. 32 (1965), 187-204.

4. P. L. Duren, Theory of $H^{p}$ space, Academic Press, New York, 1970.

5. V. K. Dzjadyk, An introduction to uniform approximation to functions by polynomials, Nauka, Moscow, 1977.

6. D. Gaier, Über Interpolation in regelmässig verteilten Punkten mit Nebenbedinguner, Math. Z. 61 (1954), 119-133.

7. ___ Vorlesungen über Approximation im Komplexen, Birkhauser-Verlag, Basel, 1980.

8. Xie-Chang Shen, On the order of approximation by Hermite-Fejér polynomials in a Jordan domain, Approximation Theory VI, Academic Press, New York, 1989.

9. roots of unity, Chinese Ann.. Math. (to appear).

10. Xie-Chang Shen and Le-Fan Zhong, Approximation in the mean by Lagrange interpolating polynomials in the complex plane, Kuxue Tongbao (A Monthly of Sciences) 33 (1988), 810-814.

11. J. Szabados and A. K. Varma, On an open problem of $P$. Turán concerning Birkhoff interpolation on the roots of unity, J. Approx. Theory 47 (1986), 255-264. 
12. A. K. Varma, Complex interpolating polynomials, Proc. Amer. Math. Soc. 103 (1988), 125-130.

13. P. Vertesi, Linear operator on the roots of unity, Studia Sci. Math. Hungar. 15 (1980), 241-245.

14. C. M. Wu, The boundary properties of conformal mapping, Acta Math. Sinica 7 (1957), $271-276$.

15. A. Zygmund, Trigonometric series, Cambridge Univ. Press, Cambridge, 1968.

Department of Mathematics, Texas A\&M University, College Station, Texas 77843

Institute of Mathematics, Peking University, People's Republic of China 